\title{
Studies of Higgs boson production in the four-lepton final state at $\sqrt{s}=13 \mathrm{TeV}$ on CMS detector
}

\author{
Tongguang Cheng*t \\ IHEP, CAS, Beijing \\ E-mail: tongguang. cheng@cern.ch
}

Studies of Higgs boson production are presented using the $\mathrm{H} \rightarrow \mathrm{ZZ} \rightarrow 4 \ell(\ell=\mathrm{e}, \mu)$ decay channel. These studies are performed using a data sample corresponding to an integrated luminosity of $2.8 \mathrm{fb}^{-1}$ of pp collisions at a center-of-mass energy of $13 \mathrm{TeV}$ collected by the CMS experiment at the LHC during 2015. The observed significance for the standard model Higgs boson with $m_{\mathrm{H}}=125.09 \mathrm{GeV}$ is $2.5 \sigma$, where the expected significance is $3.4 \sigma$. The model independent fiducial cross section is measured to be $\sigma_{\text {fid. }}=2.48_{-1.14}^{+1.48}$ (stat. $\oplus$ sys. $)_{-0.04}^{+0.01}$ (model dep.) fb. In addition, a search for an additional Higgs boson is performed for a range of masses up to $1 \mathrm{TeV}$ and with various widths, and no significant excess is observed. The results of this search are interpreted in the context of the two Higgs doublet model.

Fourth Annual Large Hadron Collider Physics

13-18 June 2016

Lund, Sweden

${ }^{*}$ Speaker.

${ }^{\dagger}$ On behalf of CMS collaboration 


\section{Introduction}

The start of the LHC Run 2 in 2015, at an increased center-of-mass energy of $\sqrt{s}=13 \mathrm{TeV}$, opens the way for an era of new precision measurements of the Higgs boson. This note presents the first CMS studies of Higgs boson production in the $\mathrm{H} \rightarrow 4 \ell$ decay channel at $\sqrt{s}=13 \mathrm{TeV}$, using $2.8 \mathrm{fb}^{-1}$ of proton-proton collision data collected with the CMS experiment at the LHC in 2015. Detail of the studies can be found in Ref. [1] with references therein.

\section{Background estimation}

The irreducible background to the Higgs signal in the $4 \ell$ channel comes from the production of $\mathrm{ZZ}$ via $\mathrm{q} \overline{\mathrm{q}}$ annihilation or gluon fusion. The contribution from these backgrounds are estimated using simulation. Higher order QCD and electroweak corrections are applied by K factors as a function of four-lepton mass.

Reducible backgrounds arise from $Z+$ jets and $t \bar{t}$ production where heavy-flavor jets produce secondary leptons, and from $\mathrm{Z}+$ jets, $\mathrm{Z} \gamma+$ jets, $\mathrm{WW}+$ jets and $\mathrm{WZ}+$ jets events where decays of heavy-flavor hadrons, in-flight decays of light mesons within jets, or (for electrons) the decay of charged hadrons overlapping with $\pi^{0}$ decays are misidentified as leptons. Two independent control regions in data are formed by requiring the presence of two leptons which satisfy the full identification criteria, plus two additional opposite-sign or same-sign leptons satisfying relaxed identification requirements. The event yields in the control regions are weighted by the lepton misidentification probability to obtain the expected yield in the signal region.

\section{Signal modeling}

The signal lineshape of a narrow resonance around $m_{\mathrm{H}} \sim 125 \mathrm{GeV}$ and mass resolution for higher-mass resonances are parametrized using a double-sided Crystal Ball function. In addition, a Landau function is also added in the total probability density function for the non-resonant part of the signal for the case of $\mathrm{WH}, \mathrm{ZH}$ and $\mathrm{t} \mathrm{t} H$ production modes. In the case of a search for an additional high-mass resonance, a general parameterization of the signal and background $\mathrm{gg} \rightarrow 4 \ell$ process is used which includes all contributing amplitudes for $\mathrm{gg} \rightarrow \mathrm{bkg}+\mathrm{H}(125)^{*}+\mathrm{X}\left(m_{X}\right) \rightarrow 4 \ell$, and their interference.

\section{Results}

\subsection{Significance and signal strength}

Figure 1 (left) shows the significance of the local fluctuation with respect to the SM backgroundonly expectation as a function of $m_{\mathrm{H}}$. The minimum of the local $p$-value is reached at $123.4 \mathrm{GeV}$ and corresponds to a local significance of $3.0 \sigma$, while $3.1 \sigma$ are expected for the SM Higgs boson. Two signal-strength modifiers $\mu_{\mathrm{ggH}, \mathrm{tt} H}$ and $\mu_{\mathrm{VBF}, \mathrm{VH}}$ are defined as scale factors for the fermion and vector-boson induced contributions to the expected SM cross section. A two-dimensional fit is performed assuming a mass of $m_{\mathrm{H}}=125.09 \mathrm{GeV}$ and profiling the likelihood for all nuisance parameters, leading to the measurements of $\mu_{\mathrm{ggH}, \mathrm{ti \textrm {H }}}=0.95_{-0.49}^{+0.64}$ and $\mu_{\mathrm{VBF}, \mathrm{VH}}=0.0_{-0.0}^{+2.5}$. The $68 \%$ and $95 \%$ CL contours in the $\left(\mu_{\mathrm{ggH}, \mathrm{tt} H}, \mu_{\mathrm{VBF}, \mathrm{VH}}\right)$ plane are shown in Fig.1 (right). 

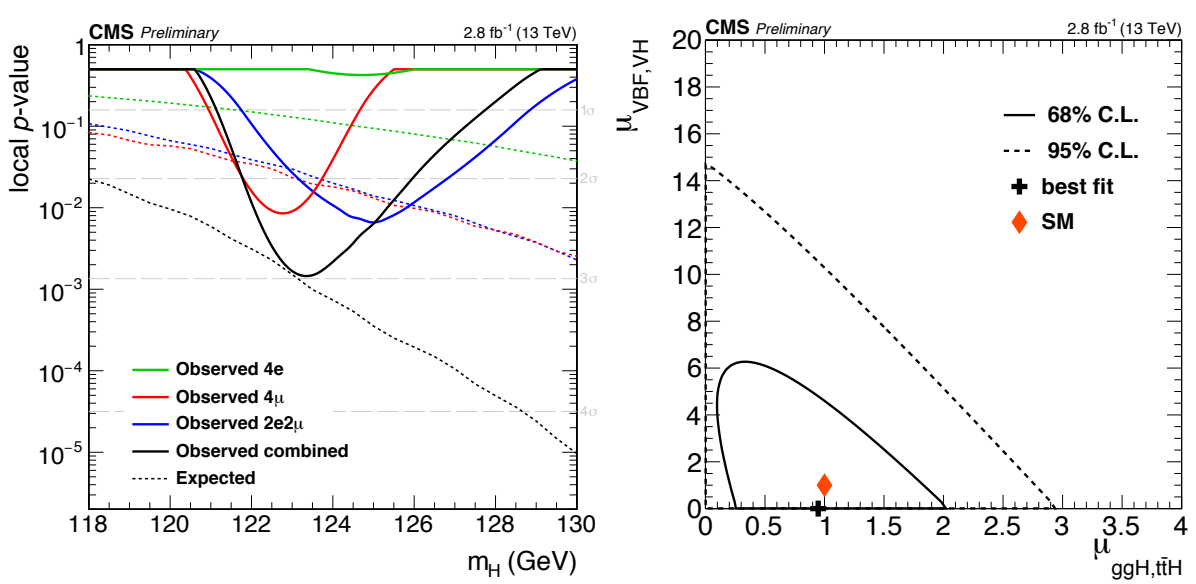

Figure 1: (left) Significance of the local fluctuation with respect to the background-only expectation as a function of the Higgs boson mass. (right) Result of the 2D likelihood scan for the $\mu_{\mathrm{ggH}, \mathrm{ttH}}$ and $\mu_{\mathrm{VBF}, \mathrm{VH}}$ signal-strength modifiers. The solid and dashed contours show the 68\% and 95\% CL regions, respectively.

\subsection{Fiducial cross section}

The fiducial volume for the cross section measurement is defined to match closely the reconstruction level selection and is very similar to the definition used in Ref. [2]. The only difference in the fiducial volume definition with respect to the Run 1 analysis is that the leptons are dressed by adding the four-momenta of photons within $\Delta R<0.4$ to the bare leptons. The integrated fiducial cross section is measured to be $\sigma_{\text {fid. }}=2.48_{-1.13}^{+1.46}$ (stat. $)_{-0.18}^{+0.28}$ (sys. $)_{-0.04}^{+0.01}$ (model dep.) fb. This can be compared to the $\mathrm{SM}$ expectation $\sigma_{\text {fid. }}^{\mathrm{SM}}=2.39 \pm 0.25 \mathrm{fb}$. The integrated fiducial cross section as a function of $\sqrt{s}$ is also shown in Fig. 2 (right).
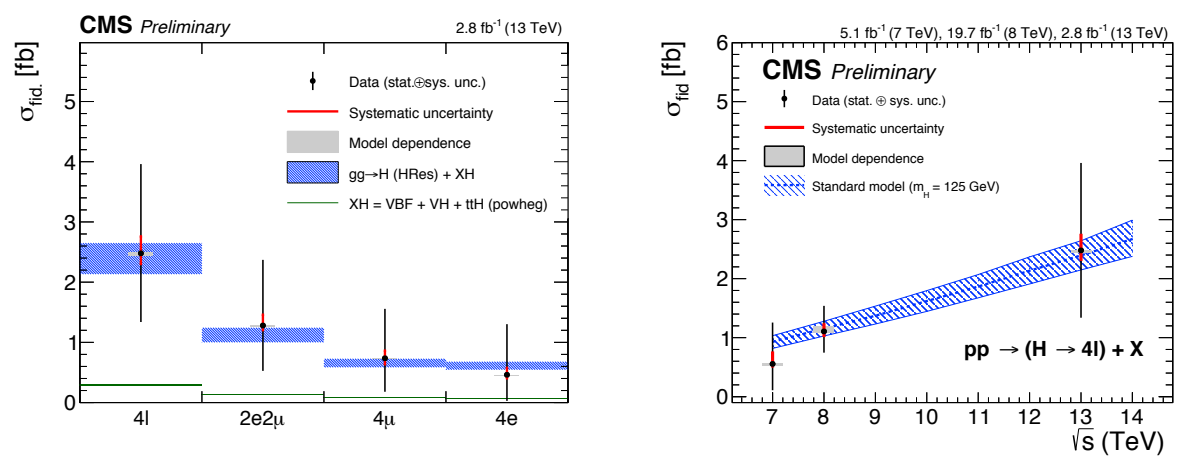

Figure 2: Result of the measured fiducial cross section in each final state (left) and the measured fiducial cross section as a function of $\sqrt{s}$ (right).

\subsection{Search for additional resonances}

Figure 3 shows observed and expected 95\% CL limits on the $\mathrm{gg} \rightarrow \mathrm{X} \rightarrow \mathrm{ZZ} \rightarrow 4 \ell$ cross section including the four-lepton branching fraction, as a function of $m_{X}$, for several values of $\Gamma_{X}$. 
In addition to the generic cross section limit, the results are also interpreted in the context of a two Higgs doublet model (2HDM) benchmark scenario. The parameters $m_{\mathrm{H}}$, the mass of the heaviest CP-even Higgs boson, and $\tan \beta$ are chosen for a two-dimensional scan. The results are shown in Fig. 3.

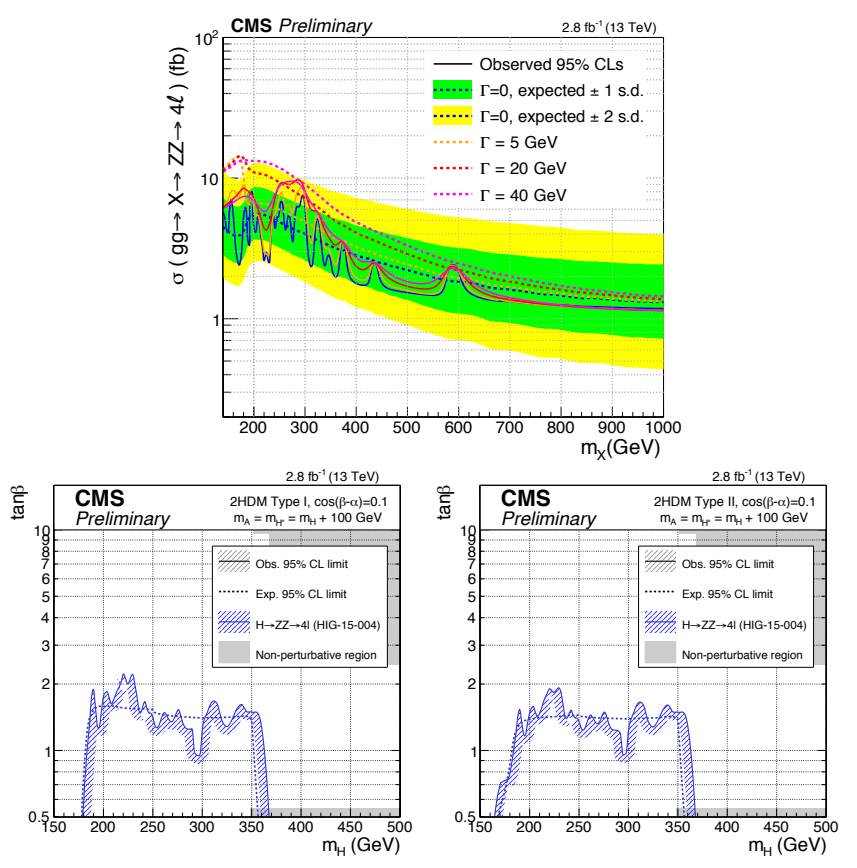

Figure 3: Top: Upper limits at the 95\% $\mathrm{CL}$ on the $\mathrm{gg} \rightarrow \mathrm{X} \rightarrow \mathrm{ZZ} \rightarrow 4 \ell$ cross section $\sigma$, including four-lepton branching fraction, as a function of $m_{\mathrm{X}}$, shown for several values of $\Gamma_{\mathrm{X}}$. Bottom: Exclusion limits at the 95\% CL in the $m_{\mathrm{H}}-\tan \beta$ plane for the benchmark 2HDM scenario with Type I (left) and Type II (right) couplings.

\section{Summary}

Several studies of Higgs boson production in the four-lepton final state at $\sqrt{s}=13 \mathrm{TeV}$ have been presented, using data samples corresponding to an integrated luminosity of $2.8 \mathrm{fb}^{-1}$. The observed significance for the SM Higgs boson at a mass of $m_{\mathrm{H}}=125.09 \mathrm{GeV}$ is $2.5 \sigma$. The measured signal strength modifiers and the model-independent fiducial cross section for this boson are consistent, within their uncertainties, with the expectations for the SM Higgs boson. In addition, upper limits at a 95\% CL are set on the production of an additional Higgs boson for masses up to $1 \mathrm{TeV}$ and for various widths, and these results are interpreted in two 2HDM scenarios.

\section{References}

[1] CMS Collaboration, CMS Physics Analysis Summary Report No. CMS-PAS-HIG-15-004, 2015. [http://cdsweb.cern.ch/record/2139978].

[2] CMS Collaboration, "Measurement of differential cross sections for Higgs boson production in the diphoton decay channel in pp collisions at $\sqrt{s}=8$ TeV", Eur. Phys. J. C 76 (2015) 13 